\title{
Mitochondrial roles of the psychiatric disease risk factor DISC1
}

Norkett, R. Modi, S. Kittler, J.T.

Department of Neuroscience, Physiology and Pharmacology, University College London, Gower Street London, UK

\section{Abstract}

Ion transport during neuronal signalling utilizes the majority of the brain's energy supply. Mitochondria are key sites for energy provision through ATP synthesis and play other important roles including calcium buffering. Thus, tightly regulated distribution and function of these organelles throughout the intricate architecture of the neuron is essential for normal synaptic communication. Therefore, delineating mechanisms coordinating mitochondrial transport and function is essential for understanding nervous system physiology and pathology. While aberrant mitochondrial transport and dynamics have long been associated with neurodegenerative disease, they have also more recently been linked to major mental illness including schizophrenia, autism and depression. However, the underlying mechanisms have yet to be elucidated, due to an incomplete understanding of the combinations of genetic and environmental factors contributing to these conditions. Consequently, the DISC1 gene has undergone intense study since its discovery at the site of a balanced chromosomal translocation, segregating with mental illness in a Scottish pedigree. The precise molecular functions of DISC1 remain elusive. Reported functions of DISC1 include regulation of intracellular signalling pathways, neuronal migration and dendritic development. Intriguingly, a role for DISC1 in mitochondrial homeostasis and transport is fast emerging. Therefore, a major function of DISC1 in regulating mitochondrial distribution, ATP synthesis and calcium buffering may be disrupted in psychiatric disease. In this review, we discuss the links between DISC1 and mitochondria, considering both trafficking of these organelles and their function, and how, via these processes, DISC1 may contribute to the regulation of neuronal behavior in normal and psychiatric disease states. 


\section{Keywords}

DISC1, Miro1, Mitochondria, Mitochondrial trafficking, Mitochondrial dynamics, synapse

\section{Abbreviations}

DISC1 - disrupted in schizophrenia 1, Miro - Mitochondrial Rho GTPase, ATP Adenosine triphosphate, OXPHOS - oxidative phosphorylation, KIF - Kinesin family, TRAK - Trafficking kinesin binding protein, OMM - Outer mitochondrial membrane, IMM - Inner mitochondrial membrane

\section{Introduction}

Disrupted in schizophrenia 1 - DISC1 - was first identified as a candidate susceptibility factor for psychiatric disease in a Scottish pedigree. In this study, it was found that a balanced chromosomal translocation co-segregated with psychiatric diagnoses including schizophrenia, bipolar disorder and major depression (St Clair et al., 1990). Analysis of the interrupted regions on these chromosomes (1 and 11) led to the discovery of the DISC1 gene on chromosome 1, encoding a protein proposed to have a large, globular, $\mathrm{N}$ terminus and a coiled coil rich $\mathrm{C}$ terminus (Millar et al., 2000). The gene on chromosome 11 was termed Boymaw or DISC1FP1 for DISC1 fusion partner 1. Figure 1a shows a schematic of the DISC1 protein structure with the position of psychiatric disease associated variants marked. The balanced chromosomal translocation has been proposed to have three potential outcomes. It may give rise to abnormal transcripts encoding DISC1 $1-597$ plus 60 or 69 novel amino acids derived from the gene on chromosome 11 (known as DISC1-Boymaw fusion protein or CP60/69 for chimaeric protein 60 or 69 ) or a truncated DISC1 transcript, ending at the break point (Brandon and Sawa, 2011). Alternatively, the abnormal transcript may not be expressed, giving rise to a haploinsufficiency 
(Brandon and Sawa, 2011) (Figure 1b). Beyond the balanced chromosomal translocation, multiple other linkage analyses and sequence analyses have proposed DISC1 as a risk factor in psychiatric illness. For example, polymorphisms in DISC1 such as a 4-base pair deletion, or point mutations giving rise to amino acid substitutions R37W, S704C and L607F segregate with schizophrenia and other major mental illness (Callicott et al., 2005; Hodgkinson et al., 2004; Sachs et al., 2005; Thomson et al., 2014). The 4-base pair deletion results in the loss of $46 \mathrm{C}$ terminal amino acids of wild type DISC1, and instead, the inclusion of 9 novel amino acids (Sachs et al., 2005). From work in patient derived neurons, we now know this transcript to be expressed at the protein level and to contribute to a defect in presynaptic function - perhaps via increased turnover of wildtype DISC1 (Wen et al., 2014). R37W and L607F have been described to impair mitochondrial trafficking, to be discussed below (Atkin et al., 2011; Ogawa et al., 2014). Further work into the functional effects of these, and other, mutations remains to be carried out, but, at the biochemical level, DISC1 is linked to sporadic schizophrenia. It has been shown to form insoluble aggregates in post mortem schizophrenia tissue, perhaps induced by cellular stress (Leliveld et al., 2008). It should be noted that DISC1 fails to appear as a genetic risk factor in genome wide association studies (International Schizophrenia et al., 2009; O'Donovan et al., 2008). However, DISC1 is known to influence endophenotypes associated with psychiatric disease such as cortical thickness and anhedonia (Callicott et al., 2005; Tomppo et al., 2009). Thus, thorough analysis of DISC1 function could give rise to deeper understanding of pathways interrupted in these diseases.

Since its discovery, the function of DISC1 in the neuron has undergone intense study, revealing roles in intracellular trafficking, neuronal development and intracellular signalling. Yeast two hybrid studies have suggested over 100 potential interaction partners (Brandon and Sawa, 2011; Camargo et al., 2007). These include kinesin motor proteins (Taya et al., 2007; Tsuboi et al., 2015), and dynein motor complex components Lis1, Nde1 and Ndel1 (Kamiya et al., 2006). Interaction with components of molecular motor complexes highlights potential roles in intracellular trafficking (see (Devine et al., 2016b) for a more detailed review). Interaction with Nde1/Ndel1 has led to elucidation of DISC1 as a regulator of neuronal differentiation, migration and integration during neuronal development (Brandon and Sawa, 2011; 
Duan et al., 2007; Enomoto et al., 2009; Narayan et al., 2013; Singh et al., 2011). Further, interactions with GSK3 beta and phosphodiesterases suggest roles in intracellular signalling (Ishizuka et al., 2011; Millar et al., 2005b). Beyond these functions, DISC1 has been localised to mitochondria in multiple studies via electron microscopy, immunocytochemistry and biochemical fractionation experiments (James et al., 2004; Millar et al., 2005a; Norkett et al., 2016; Park et al., 2010) and found to interact with mitochondrial proteins mitofilin and $\mathrm{CHCHD} 6$ - which influence mitochondrial function (Park et al., 2010; Pinero-Martos et al., 2016). Along with its interaction with trafficking machinery this has suggested DISC1 as a crucial regulator of mitochondrial trafficking and function which will be further discussed below.

Mitochondria are considered as the powerhouses of cells, generating ATP to facilitate key cellular functions such as ion pumping, cellular trafficking, and neuronal communication. They are comprised of an outer membrane, inter membrane space and highly selective inner mitochondrial membrane, preventing free diffusion of ions and small molecules, thus compartmentalising the matrix from the cytosol (see figure 2a). This inner membrane is intricately folded into cristae to increase surface area and is the site of oxidative phosphorylation for ATP production. The inner membrane surrounds the mitochondrial matrix (Cogliati et al., 2016). Besides ATP production, mitochondria are known to buffer $\mathrm{Ca}^{2+}$ during release of neurotransmitters or postsynaptic receptor activation which, in turn, regulates neural signalling (McBride et al., 2006; Sheng and Cai, 2012; Szabadkai and Duchen, 2008; Werth and Thayer, 1994; Zucker, 1999). In order to achieve this, mitochondria must be precisely situated within neuronal processes at sites of high ATP or calcium buffering demand, such as the pre and post synapse. Therefore, mitochondria must be trafficked to, and docked at these sites to allow correct neuronal function and development (MacAskill et al., 2010; MacAskill and Kittler, 2010).

Several studies have demonstrated that physical proximity between mitochondria and synapses is dependent on neuronal activity (Courchet et al., 2013; Macaskill et al., 2009b; Sheng and Cai, 2012). Neural activity consumes energy in terms of ATP which is provided by the mitochondria. Studies suggested that ATP derived from mitochondria is important for long-term potentiation (LTP) and dendritic spine morphogenesis (Li et al., 2004; Lowe et al., 2013). Synaptic plasticity has a key role 
in both short- and long-term memory and is often linked to a variety of psychiatric disorders (Takeuchi et al., 2013; Wondolowski and Dickman, 2013). Close proximity to synapses enables mitochondria to buffer $\mathrm{Ca}^{2+}$, which plays an important role in neurotransmission at central synapses. Beyond spine morphogenesis, mitochondrial distribution is essential for correct neurite outgrowth, and thus development of the neuron and circuit connectivity (Chen et al., 2007; Fukumitsu et al., 2015; Norkett et al., 2016). Moreover, mitochondrial distribution is linked to neuronal differentiation and migration (Kim et al., 2015; Lin-Hendel et al., 2016). These processes are also well recognised to be influenced by DISC1 function, and known to be impaired in psychiatric disease (Brandon and Sawa, 2011; International Schizophrenia et al., 2009).

In addition to the trafficking and positioning of mitochondria, other aspects of mitochondrial function such as mitochondrial $\mathrm{Ca}^{2+}$ uptake or release, the production of superoxide and other ROS (reactive oxygen species) are also increasingly implicated in synaptic plasticity (Cheng et al., 2010; Levy et al., 2003; Santini et al., 2015; Vos, 2010). Interestingly, calcium overload or oxidative stress can significantly impair mitochondrial function and this form of dysfunction in specific brain regions are often correlated with major depressive disorder (Lin and Beal, 2006; Nicholls, 2009; Shao et al., 2008). Whilst psychiatric diseases do not present with classical mitochondrial dysfunction, as do multiple neurodegenerative disease including Alzheimer's disease, Parkinson's disease and Huntington's disease (Carvalho et al., 2015), mitochondria are essential to power and regulate neuronal development and function. Thus, there has been an effort to investigate the contribution of these organelles to the onset of major mental illness (as reviewed in (Clay et al., 2011; Deheshi et al., 2013; Manji et al., 2012; Rossignol and Frye, 2012)). Evidence for mitochondrial abnormalities has been described in post mortem tissue from patients with psychiatric disease. Electron microscopy has revealed a reduction in the number and density of mitochondria in the hippocampus, striatum and substantia nigra of schizophrenia patients (Kolomeets and Uranova, 2010; Kung and Roberts, 1999; Roberts et al., 2015). 


\section{Localisation of DISC1 at the mitochondrion}

DISC1 is a multi-compartmentalised protein, which can localise to mitochondria as demonstrated by subcellular fractionation, immunocytochemistry and electron microscopy (James et al., 2004; Millar et al., 2005a; Millar et al., 2005b; Norkett et al., 2016; Ogawa et al., 2014; Park et al., 2010). It would seem this targeting is dependent upon the $\mathrm{N}$ terminus of DISC1 (Millar et al., 2005a). Immunocytochemistry shows a predominantly mitochondrial localisation in two neuroblastoma cell lines (U373 MG and SH-SY5Y), although puncta are also detected both in the periphery and the nucleus (James et al., 2004). Further studies in cortical neurons are consistent with this heterogeneous distribution of DISC1 (Brandon et al., 2005; Park et al., 2010). DISC1 lacks a classic mitochondrial targeting sequence, therefore other factors must allow this mitochondrial targeting, such as interaction with mitochondrial proteins. Those that have been studied in this way include trafficking proteins, Miro1, Miro2 and TRAK1 and TRAK2, and the mitochondrial fusion machinery Mitofusins, (Norkett et al., 2016; Ogawa et al., 2014; Park et al., 2010)- overexpression of each showing a marked redistribution of DISC1 to the mitochondria. Further, two studies show a redistribution of DISC1 away from mitochondria and into the cytosolic fraction upon destabilisation of microtubules with taxol or nocodazole (Brandon et al., 2005; James et al., 2004). These observations represent early indications of a role for DISC1 in microtubule based transport of mitochondria.

In addition to the outer mitochondrial membrane, ultrastructural and biochemical analyses have demonstrated the presence of both exogenous and endogenous DISC1 inside mitochondria in HEK cells and mouse brain (Park et al., 2010). Treatment of mitochondrial fractions with trypsin has shown specific DISC1 isoforms to be protected, while outer mitochondrial membrane proteins are digested, supportive of localisation at internal mitochondrial compartments (James et al., 2004; Park et al., 2010). Further investigation of localisation of multiple DISC1 isoforms has revealed the longer, $100 \mathrm{kDa}$ isoforms are degraded by this treatment, consistent with an outer mitochondrial membrane distribution whereas, the shorter, 71 and $75 \mathrm{kDa}$ isoforms were protected (James et al., 2004; Pinero-Martos et al., 2016). 
The effects of schizophrenia associated DISC1 mutations on mitochondrial distribution of the protein are less clear. It has been shown that an R37W mutation leads to a more uniform distribution of DISC1 on the mitochondria, and induces mitochondrial clustering. In neurons, this mutation seems to increase colocalisation of DISC1 with the mitochondria (Ogawa et al., 2014). Further, the DISC1-Boymaw fusion protein has been shown to localise to the mitochondria to a greater extent than the wild type protein (Eykelenboom et al., 2012; Norkett et al., 2016). This altered distribution of mutant DISC1 confers defects in mitochondrial trafficking and function to be discussed in the following sections.

\subsection{DISC1 and mitochondrial function - ATP production}

The presence of DISC1 both on the mitochondrial outer membrane and within internal compartments raises the possibility that DISC1 could act within the mitochondrion to regulate function of these organelles. Further, two independent yeast two hybrid screens have identified the inner mitochondrial membrane protein Mitofilin as a DISC1 interactor (Camargo et al., 2007; Park et al., 2010). Interaction with this protein has been confirmed at the protein level (Park et al., 2010) and is of particular interest as Mitofilin (also known as IMMT for inner membrane protein, mitochondrial) has been shown to regulate cristae formation as part of the mitochondrial contact site and cristae organizing system (MICOS) complex (John et al., 2005). These cristae (in-foldings) in the inner mitochondrial membrane serve as the site of ATP production via oxidative phosphorylation (OXPHOS). Mitofilin knockdown studies in HeLa cells showed less organised cristae, associated with an increase in reactive oxygen species (John et al., 2005). Thus, Mitofilin regulates mitochondrial function in terms of ATP production. The interaction between DISC1 and Mitofilin has been localised to the mitochondrial fraction of cell lysates and multiple experiments were carried out to investigate crosstalk of these two proteins in regulating mitochondrial function (see figure 2a). Knockdown of DISC1 both decreased NADH dehydrogenase activity and ATP production (Park et al., 2010; Pinero-Martos et al., 2016). These effects mimicked those obtained from Mitofilin knockdown cells. Further, expression of truncated DISC1 (amino acids 1-597, resulting from the Scottish mutation) also caused a decrease in these measures (Park et al., 2010). This phenocopy is likely due to the necessity of DISC1 in 
stabilising the Mitofilin protein. Knockdown or expression of truncated DISC1 causes an increase in Mitofilin ubiquitination and subsequent proteasomal turnover (Park et al., 2010). Thus, DISC1 acts as a regulator of mitochondrial function via Mitofilin levels (see figure 2b).

In addition to DISC1, another of its interaction partners, CHCHD6 - a MICOS component - has been shown to regulate Mitofilin levels. This protein is localised to the inner mitochondrial membrane according to fractionation experiments and knockdown of this protein has similar, adverse, effects on cristae organisation as does Mitofilin. As with Mitofilin, knockdown of CHCHD6 leads to a decrease in mitochondrial ATP production (An et al., 2012). This raises the possibility that DISC1, Mitofilin and CHCHD6 exist together in a large complex involved in mitochondrial function by maintaining cristae integrity. Indeed, DISC1 knockdown leads to disassembly of the cristae organisation complex as well disassembly of OXPHOS complexes (see figure $2 \mathrm{~b}$ ), as demonstrated by blue native PAGE (PineroMartos et al., 2016), suggesting DISC1 may be acting as a scaffold for assembly of cristae organisation complexes, thus facilitating OXPHOS.

As well as DISC1 truncation, alternative outcomes of the Scottish mutation have been investigated with a focus on mitochondrial dysfunction (Eykelenboom et al., 2012). The distribution of DISC1-Boymaw fusion transcripts was investigated in COS7 cells and primary neurons. In both cases, the novel transcripts adopted a predominantly mitochondrial localisation, in contrast to truncated DISC1 1-597, which appeared diffuse throughout the cytosol. Thus, it appears the novel amino acids confer a gain of function in terms of distribution. It was notable that mitochondria of cells expressing fusion transcripts showed decreased mitotracker staining in comparison to cytochrome $C$ labelling. This observation might suggest that the novel transcripts decrease mitochondrial membrane potential, as mitotracker is selectively taken up into functional mitochondria (Eykelenboom et al., 2012). Thus it would appear that DISC1-Boymaw fusion transcripts have deleterious effects on mitochondrial function, independent of wildtype DISC1, although endogenous DISC1 levels were not investigated in cells heterologously expressing the chimaeric proteins. Therefore, indirect effects via loss of DISC1 expression cannot be ruled out. 
Consistent with these observations, the DISC1-Boymaw fusion protein (CP60 by the alternative nomenclature), was shown to localise to mitochondria in two recent studies (Ji et al., 2014; Norkett et al., 2016). Additionally, a decrease in NADH oxidoreductase activity was detected in total lysates and mitochondrial fractions of cells expressing the DISC1-Boymaw fusion protein. This may contribute to a decrease in ATP production via mitochondrial damage (Ji et al., 2014). The Boymaw gene itself has been recently suggested to encode a small protein (Ji et al., 2015). This is in contrast with earlier reports which suggested no open readings frames were present in this gene (Eykelenboom et al., 2012; Zhou et al., 2010; Zhou et al., 2008). When overexpressed in HEK293 cells, this small Boymaw protein localises to mitochondria and inhibits NADH oxidoreductase activity (Ji et al., 2015). Taken together, these data are supportive of mitochondrial damage, caused by the DISC1Boymaw fusion protein, being a pathological feature of schizophrenia.

\subsection{Calcium Buffering}

In addition to ATP production, DISC1 may influence mitochondrial calcium buffering. Upon DISC1 or Mitofilin knockdown, mitochondria were shown to buffer calcium more slowly than control cells using a fluorescent calcium reporter (GCAMP) in differentiating CAD cells. This was exemplified by an increase in the time taken for intra mitochondrial calcium levels to return to baseline after ionomycin treatment. Therefore, DISC1, in conjunction with Mitofilin, can regulate both the bioenergetic and calcium buffering capabilities of mitochondria (Park et al., 2010).

Beyond mitochondrial calcium handling, DISC1 has also been localised to the ER and shown to interact with the $\mathrm{IP}_{3}$ receptor - a component of ER-mitochondria contact sites (Csordas et al., 2010; Park et al., 2015). In addition to the $\mathrm{IP}_{3}$ receptor, DISC1 interacts with Miro (Norkett et al., 2016; Ogawa et al., 2014)- itself shown to be a component of ER-mitochondria sites in Saccharomyces and Drosophila (Kornmann et al., 2011; Lee et al., 2016). In neurons cultured from a DISC1 knockout mouse model, ER calcium dynamics were shown to be perturbed with the knock out cells displaying higher calcium uptake than wild type - a phenotype which could be reversed by antipsychotics (Park et al., 2015). This dual action of DISC1 in contributing to calcium dynamics in both mitochondrial and ER compartments raises 
the possibility that DISC1 might coordinate crosstalk of these organelles via ER mitochondria contact sites. Indeed, super resolution microscopy studies have found DISC1 to be present at these sites (Norkett et al., 2016). Furthermore, when comparing distributions of wild type DISC1 and a schizophrenia associated DISC1 mutant (the DISC1-Boymaw fusion protein), it appears the mutant DISC1 localises to these sites more than the wild type. Crucially, this mutant form of DISC1 decreased the area of mitochondria-ER contact (Norkett et al., 2016). Thus, dysregulation of ER-mitochondria calcium signalling could be a contributory factor in the onset of psychiatric disease.

\section{DISC1 as a regulator of mitochondrial trafficking}

Beyond mitochondrial function, there are many lines of evidence supporting DISC1 as a mitochondrial trafficking regulator. In order to properly distribute mitochondria throughout the neuron, the mitochondrial population is trafficked in both anterograde and retrograde directions along microtubule tracks using motor proteins and adaptors found on mitochondrial surfaces (MacAskill and Kittler, 2010). The motor proteins involved are Kinesins (specifically Kinesin-1/KIF5 family members and KIF1Bbeta) for anterograde trafficking (towards the microtubule plus end) and Dynein (towards the microtubule minus end) (Macaskill et al., 2009b; Nangaku et al., 1994; Pilling et al., 2006).

Major players of this trafficking are Miro proteins, or Mitochondrial Rho-GTPases, anchored into the outer mitochondrial membrane, facing the cytosol. The Miro GTPases tether mitochondria to motor proteins and adaptors to enable long range transport in dendrites and axons (Birsa et al., 2013; Devine et al., 2016a; LopezDomenech et al., 2016; Macaskill et al., 2009b; Wang and Schwarz, 2009). The actin cytoskeleton and different myosin motors may also be involved in short range trafficking of this dynamic organelle (Quintero et al., 2009). Adaptor proteins involved include TRAK1 and TRAK2 (Milton in Drosophila Melanogaster). The TRAKS have been shown to form complexes with mammalian Miro proteins and indirectly regulate mitochondrial trafficking in both axons and dendrites (Brickley and Stephenson, 2011; MacAskill et al., 2009a; van Spronsen et al., 2013). 
Multiple studies have confirmed protein-protein interactions between DISC1 and components of molecular motor complexes. These proteins include the molecular motors themselves kinesin-1 family members and the adaptor proteins TRAK1 and 2 (Norkett et al., 2016; Ogawa et al., 2014; Taya et al., 2007). Moreover, DISC1 interactions have been shown with dynein complex proteins Lis1 and Ndel1 (Kamiya et al., 2005), required for dynein based, retrograde trafficking of mitochondria (Shao et al., 2013). Recently, DISC1 interactor and dynein complex component Nde1 has been shown to associate with TRAK1 in a phospho-dependent manner (Ogawa et al., 2016). This phosphorylation is itself dependent upon DISC1 via interaction with phosphodiesterase 4 (Bradshaw et al., 2011). Crucially, immunoprecipitation experiments have demonstrated interactions between DISC1, Miro1 and TRAKs in cell lysates and brain tissue (Norkett et al., 2016; Ogawa et al., 2014). These interactions have been mapped onto DISC1 both biochemically and by immunofluorescence. Immunoprecipitation experiments with DISC1 deletion constructs and Miro/TRAK show the globular N-terminus to be necessary for interaction with Miro1 and TRAK, whilst the coiled-coil rich C-terminus is dispensable. Within the $\mathrm{N}$-terminus, an arginine rich sequence has been shown to mediate the DISC1-TRAK interaction - deletion or mutation of this sequence decreased the DISC1-TRAK interaction and decreased mitochondrial DISC1 (Norkett et al., 2016; Ogawa et al., 2014). Importantly, the DISC1-Miro/TRAK interaction has been shown to be essential for mitochondrial transport. Overexpression of the interacting region of DISC1 (to uncouple endogenous DISC1 from the mitochondrial trafficking complex) abrogates mitochondrial trafficking in neuronal culture (Norkett et al., 2016) and expression of a R37W mutant transcript abolishes the ability of DISC1 to promote anterograde mitochondrial transport, perhaps via increased association of DISC1 and TRAK1, preventing the TRAK1-Miro interaction (Ogawa et al., 2014). The interaction of DISC1 with this mitochondrially anchored protein confers specificity for mitochondrial trafficking over regulation of trafficking complexes in general, and highlights the ability of DISC1 to control mitochondrial motility locally at the trafficking complex (see figure $3 a$ ).

Of further interest, the roles of schizophrenia associated DISC1 mutations have been investigated with respect to mitochondrial trafficking. Upon knockdown of DISC1, expression of S704C was able to restore percentages of motile mitochondria to their 
control levels. However, another variant, L607F, was unable to rescue this effect (Atkin et al., 2011). Overexpression of the DISC1 point mutation R37W appears to cause a decrease in anterograde (kinesin mediated) mitochondrial trafficking in comparison to overexpression of wildtype DISC1 (Ogawa et al., 2014). As well as point mutations, the DISC1-Boymaw fusion protein (a proposed outcome of the chromosomal translocation, see figure $1 \mathrm{~b}$ ) has been shown to decrease mitochondrial trafficking in axons and dendrites, consistent with a dominant negative activity of this aberrant transcript (Norkett et al., 2016). Thus, schizophrenia associated DISC1 mutations have differing abilities to regulate mitochondrial trafficking (see figure $3 \mathrm{~b}$ ). This not only serves to highlight the role of DISC1 in mitochondrial trafficking, but shows the potential for this regulation to be lost in certain cases of schizophrenia. Thus, decreased mitochondrial motility, and aberrant distribution, may be associated with the onset of schizophrenia like symptoms, perhaps via impaired ATP production and calcium buffering at synapses. This would lead to impaired synaptic communication (Li et al., 2004). Indeed, a loss of DISC1 dependent mitochondrial transport correlates with a decrease in dendrite outgrowth (Norkett et al., 2016), which could contribute to impairments in network connectivity leading to the onset of psychiatric disease (Kulkarni and Firestein, 2012).

In addition to mutations, DISC1 aggregation has been linked to mitochondrial trafficking defects. The ability of DISC1 to aggregate has been demonstrated by its presence in sarkosyl insoluble or resistant fractions of cell lysates and is increased in schizophrenic post mortem brain tissue (Leliveld et al., 2008). Certain DISC1 mutations may confer an increase in tendency to aggregate (Trossbach et al., 2016; Wen et al., 2014; Zhou et al., 2010), but, crucially, DISC1 can aggregate in response to cell stress. Therefore, aggregation may contribute to DISC1 dysfunction in sporadic cases of schizophrenia. Further, these aggregates have been identified as aggresomes - inclusions of misfolded protein to be degraded by proteasomal and autophagic pathways. This was shown by colocalisation of GFP DISC1 with aggresome markers such as ubiquitin and HSP70. GFP tagged DISC1 has a higher propensity to aggregate and has been shown by FRAP (fluorescence recovery after photobleaching) to be stable in these aggresomal structures. Importantly, cytosolic DISC1 can be recruited to these aggregates by misfolded DISC1. This corresponds with a decrease in mitochondrial trafficking, likely by decreasing the availability of 
DISC1 as in the previously described knockdown experiments, and so contributing to the onset of psychiatric disease (Atkin et al., 2012).

Notably, the exact mechanisms by which DISC1 can regulate trafficking remain to be determined. DISC1 knockdown is known to impair mitochondrial function (Park et al., 2010), thus impaired bioenergetics could be responsible for the associated decrease in trafficking (Cai et al., 2012; Miller and Sheetz, 2004). Alternatively, DISC1 could be acting as a scaffold via interactions with molecular motors and adaptors at the outer mitochondrial membrane, for example, regulating cyclic AMP levels or GSK3beta activity to influence mitochondrial trafficking (Ogawa et al., 2016). In addition to regulating motor protein complexes, a recent study proposes that DISC1 could act as a coordinator of the interaction between Miro and the axonal mitochondrial static anchor syntaphilin (Park et al., 2016) - necessary for anchoring mitochondria at the presynapse for short term facilitation (Kang et al., 2008). In this model, DISC1 is shown to interact with the anchoring protein syntaphilin, preventing interaction between Miro and syntaphilin, thus increasing the fraction of motile mitochondria (Park et al., 2016).

\section{Outlooks}

We have described work elucidating involvement of DISC1 in aspects of mitochondrial function and distribution via interactions with components of the cristae organising complex and trafficking complex. We have also discussed how alterations in DISC1 contribute to defects in these processes either by knockdown or mutation. It remains to be confirmed if the mutations studied to date act as loss of function mutations, and thus contribute to the onset of psychiatric symptoms via a DISC1 haploinsufficiency, or if any of these mutations confer a toxic gain of function. An important aspect for future study is the connection between DISC1 involvement in mitochondrial function and involvement in trafficking. It has been shown that mitochondrial membrane potential, and so ATP production, is a positive regulator of anterograde mitochondrial trafficking (Cai et al., 2012; Miller and Sheetz, 2004). Further, calcium buffering capacity is linked both to mitochondrial trafficking and ATP production (Chang et al., 2011; McCormack et al., 1990). Calcium buffering is, in turn, facilitated by ER-mitochondria contacts (Rowland and Voeltz, 2012), another 
aspect of mitochondrial biology in which DISC1 is implicated. Might DISC1 maintain mitochondrial calcium buffering capacity and ATP production so as to facilitate trafficking? Or indeed, might multiple isoforms of DISC1 be concerned with specific functions at the mitochondrion - for example a shorter isoform within the mitochondrion, perhaps at the IMM, to regulate cristae formation and a longer isoform at the outer mitochondrial membrane to coordinate the mitochondrial trafficking complex, as suggested by fractionation studies (James et al., 2004). Further, might DISC1 play a role in maintaining the health of the mitochondrial population - and thus the health of the neuron - via involvement in the mitophagic quality control pathway? This selective degradation of damaged mitochondria is essential for neuronal health (Birsa et al., 2013; Pickrell and Youle, 2015) and Miro is a key player in this process as a substrate for the E3 ubiquitin ligase Parkin (Birsa et al., 2014; Liu et al., 2012; Wang et al., 2011). DISC1 itself can be degraded by the autophagy pathway upon aggregation (Atkin et al., 2012) and so, might contribute to impairments in mitophagy and a deleterious build-up of damaged mitochondria in psychiatric disease.

Beyond function of neurons themselves, might impaired mitochondrial trafficking in astrocytes contribute to impaired network activity? DISC1 is known to be expressed in astrocytes and other glia (Seshadri et al., 2010), and Miro has been recently demonstrated to be essential for mitochondrial positioning opposing synapses in astrocytes (Jackson and Robinson, 2015; Stephen et al., 2015). These cells are a subset of glia crucial for shaping neuronal communication at the tripartite synapse (Stephen et al., 2014). Could DISC1 be regulating mitochondrial transport in these cells, and, thus, contributing to neuronal communication? Loss of ATP production and calcium buffering capacity, via damaged or incorrectly distributed mitochondria, would impair both neuronal development and synaptic activity (Kang et al., 2008; Li et al., 2004; Norkett et al., 2016; Sung et al., 2008). These conditions would impair network connectivity, contributing to the onset of psychiatric disease. Crucially, ongoing work must address the contributions of mitochondrial aspects of DISC1 to neuronal development and function in vivo in health and mental illness. 


\section{References}

An, J., Shi, J., He, Q., Lui, K., Liu, Y., Huang, Y., Sheikh, M.S., 2012. CHCM1/CHCHD6, novel mitochondrial protein linked to regulation of mitofilin and mitochondrial cristae morphology. J. Biol. Chem. 287(10), 7411-7426.

Atkin, T.A., Brandon, N.J., Kittler, J.T., 2012. Disrupted in Schizophrenia 1 forms pathological aggresomes that disrupt its function in intracellular transport. Human molecular genetics 21(9), 2017-2028.

Atkin, T.A., Macaskill, A.F., Brandon, N.J., Kittler, J.T., 2011. Disrupted in Schizophrenia-1 regulates intracellular trafficking of mitochondria in neurons. Mol Psychiatry 16(2), 122-124.

Birsa, N., Norkett, R., Higgs, N., Lopez-Domenech, G., Kittler, J.T., 2013. Mitochondrial trafficking in neurons and the role of the Miro family of GTPase proteins. Biochem Soc Trans 41(6), 1525-1531.

Birsa, N., Norkett, R., Wauer, T., Mevissen, T.E., Wu, H.C., Foltynie, T., Bhatia, K., Hirst, W.D., Komander, D., Plun-Favreau, H., Kittler, J.T., 2014. Lysine 27 ubiquitination of the mitochondrial transport protein Miro is dependent on serine 65 of the Parkin ubiquitin ligase. J Biol Chem 289(21), 14569-14582.

Bradshaw, N.J., Soares, D.C., Carlyle, B.C., Ogawa, F., Davidson-Smith, H., Christie, S., Mackie, S., Thomson, P.A., Porteous, D.J., Millar, J.K., 2011. PKA phosphorylation of NDE1 is DISC1/PDE4 dependent and modulates its interaction with LIS1 and NDEL1. J Neurosci 31(24), 9043-9054.

Brandon, N.J., Sawa, A., 2011. Linking neurodevelopmental and synaptic theories of mental illness through DISC1. 1-16.

Brandon, N.J., Schurov, I., Camargo, L.M., Handford, E.J., Duran-Jimeniz, B., Hunt, P., Millar, J.K., Porteous, D.J., Shearman, M.S., Whiting, P.J., 2005. Subcellular targeting of DISC1 is dependent on a domain independent from the Nudel binding site. Mol Cell Neurosci 28(4), 613-624.

Brickley, K., Stephenson, F.A., 2011. Trafficking kinesin protein (TRAK)-mediated transport of mitochondria in axons of hippocampal neurons. J Biol Chem 286(20), 18079-18092.

Cai, Q., Zakaria, H.M., Simone, A., Sheng, Z.H., 2012. Spatial parkin translocation and degradation of damaged mitochondria via mitophagy in live cortical neurons. Curr Biol 22(6), 545-552.

Callicott, J.H., Straub, R.E., Pezawas, L., Egan, M.F., Mattay, V.S., Hariri, A.R., Verchinski, B.A., Meyer-Lindenberg, A., Balkissoon, R., Kolachana, B., Goldberg, T.E., Weinberger, D.R., 2005. Variation in DISC1 affects hippocampal structure and function and increases risk for schizophrenia. Proc Natl Acad Sci U S A 102(24), 8627-8632.

Camargo, L.M., Collura, V., Rain, J.C., Mizuguchi, K., Hermjakob, H., Kerrien, S., Bonnert, T.P., Whiting, P.J., Brandon, N.J., 2007. Disrupted in Schizophrenia 1 Interactome: evidence for the close connectivity of risk genes and a potential synaptic basis for schizophrenia. Mol Psychiatry 12(1), 74-86.

Carvalho, C., Correia, S.C., Cardoso, S., Placido, A.I., Candeias, E., Duarte, A.I., Moreira, P.I., 2015. The role of mitochondrial disturbances in Alzheimer, Parkinson and Huntington diseases. Expert Rev Neurother 15(8), 867-884.

Chang, K.T., Niescier, R.F., Min, K.T., 2011. Mitochondrial matrix Ca2+ as an intrinsic signal regulating mitochondrial motility in axons. Proc Natl Acad Sci U S A 108(37), 1545615461.

Chen, H., McCaffery, J.M., Chan, D.C., 2007. Mitochondrial fusion protects against neurodegeneration in the cerebellum. Cell 130(3), 548-562. 
Cheng, A., Hou, Y., Mattson, M.P., 2010. Mitochondria and neuroplasticity. ASN NEURO 2(5), 243-256.

Clay, H.B., Sillivan, S., Konradi, C., 2011. Mitochondrial dysfunction and pathology in bipolar disorder and schizophrenia. Int J Dev Neurosci 29(3), 311-324.

Cogliati, S., Enriquez, J.A., Scorrano, L., 2016. Mitochondrial Cristae: Where Beauty Meets Functionality. Trends Biochem Sci 41(3), 261-273.

Courchet, J., Lewis, T.L., Lee, S., Courchet, V., Liou, D.-Y., Aizawa, S., Polleux, F., 2013. Terminal axon branching is regulated by the LKB1-NUAK1 kinase pathway via presynaptic mitochondrial capture. Cell 153(7), 1510-1525.

Csordas, G., Varnai, P., Golenar, T., Roy, S., Purkins, G., Schneider, T.G., Balla, T., Hajnoczky, G., 2010. Imaging interorganelle contacts and local calcium dynamics at the ERmitochondrial interface. Mol Cell 39(1), 121-132.

Deheshi, S., Pasqualotto, B.A., Rintoul, G.L., 2013. Mitochondrial trafficking in neuropsychiatric diseases. Neurobiol Dis 51, 66-71.

Devine, M.J., Birsa, N., Kittler, J.T., 2016a. Miro sculpts mitochondrial dynamics in neuronal health and disease. Neurobiol Dis 90, 27-34.

Devine, M.J., Norkett, R., Kittler, J.T., 2016b. DISC1 is a coordinator of intracellular trafficking to shape neuronal development and connectivity. J Physiol 594(19), 5459-5469.

Duan, X., Chang, J.H., Ge, S., Faulkner, R.L., Kim, J.Y., Kitabatake, Y., Liu, X.-B., Yang, C.-H., Jordan, J.D., Ma, D.K., Liu, C.Y., Ganesan, S., Cheng, H.-J., Ming, G.-L., Lu, B., Song, H., 2007. Disrupted-In-Schizophrenia 1 regulates integration of newly generated neurons in the adult brain. Cell 130(6), 1146-1158.

Enomoto, A., Asai, N., Namba, T., Wang, Y., Kato, T., Tanaka, M., Tatsumi, H., Taya, S., Tsuboi, D., Kuroda, K., Kaneko, N., Sawamoto, K., Miyamoto, R., Jijiwa, M., Murakumo, Y., Sokabe, M., Seki, T., Kaibuchi, K., Takahashi, M., 2009. Roles of disrupted-inschizophrenia 1-interacting protein girdin in postnatal development of the dentate gyrus. Neuron 63(6), 774-787.

Eykelenboom, J.E., Briggs, G.J., Bradshaw, N.J., Soares, D.C., Ogawa, F., Christie, S., Malavasi, E.L., Makedonopoulou, P., Mackie, S., Malloy, M.P., Wear, M.A., Blackburn, E.A., Bramham, J., McIntosh, A.M., Blackwood, D.H., Muir, W.J., Porteous, D.J., Millar, J.K., 2012. A $t(1 ; 11)$ translocation linked to schizophrenia and affective disorders gives rise to aberrant chimeric DISC1 transcripts that encode structurally altered, deleterious mitochondrial proteins. Hum Mol Genet 21(15), 3374-3386.

Fukumitsu, K., Fujishima, K., Yoshimura, A., Wu, Y.K., Heuser, J., Kengaku, M., 2015. Synergistic action of dendritic mitochondria and creatine kinase maintains ATP homeostasis and actin dynamics in growing neuronal dendrites. J Neurosci 35(14), 5707-5723.

Hodgkinson, C.A., Goldman, D., Jaeger, J., Persaud, S., Kane, J.M., Lipsky, R.H., Malhotra, A.K., 2004. Disrupted in schizophrenia 1 (DISC1): association with schizophrenia, schizoaffective disorder, and bipolar disorder. Am J Hum Genet 75(5), 862-872.

International Schizophrenia, C., Purcell, S.M., Wray, N.R., Stone, J.L., Visscher, P.M., O'Donovan, M.C., Sullivan, P.F., Sklar, P., 2009. Common polygenic variation contributes to risk of schizophrenia and bipolar disorder. Nature 460(7256), 748-752.

Ishizuka, K., Kamiya, A., Oh, E.C., Kanki, H., Seshadri, S., Robinson, J.F., Murdoch, H., Dunlop, A.J., Kubo, K.-I., Furukori, K., Huang, B., Zeledon, M., Hayashi-Takagi, A., Okano, H., Nakajima, K., Houslay, M.D., Katsanis, N., Sawa, A., 2011. DISC1-dependent switch from progenitor proliferation to migration in the developing cortex. Nature 473(7345), 92-96. Jackson, J.G., Robinson, M.B., 2015. Reciprocal Regulation of Mitochondrial Dynamics and Calcium Signaling in Astrocyte Processes. J Neurosci 35(45), 15199-15213. 
James, R., Adams, R.R., Christie, S., Buchanan, S.R., Porteous, D.J., Millar, J.K., 2004. Disrupted in Schizophrenia 1 (DISC1) is a multicompartmentalized protein that predominantly localizes to mitochondria. Mol Cell Neurosci 26(1), 112-122.

Ji, B., Higa, K.K., Kim, M., Zhou, L., Young, J.W., Geyer, M.A., Zhou, X., 2014. Inhibition of protein translation by the DISC1-Boymaw fusion gene from a Scottish family with major psychiatric disorders. Hum Mol Genet 23(21), 5683-5705.

Ji, B., Kim, M., Higa, K.K., Zhou, X., 2015. Boymaw, overexpressed in brains with major psychiatric disorders, may encode a small protein to inhibit mitochondrial function and protein translation. American journal of medical genetics. Part B, Neuropsychiatric genetics : the official publication of the International Society of Psychiatric Genetics 168B(4), 284-295. John, G.B., Shang, Y., Li, L., Renken, C., Mannella, C.A., Selker, J.M.L., Rangell, L., Bennett, M.J., Zha, J., 2005. The mitochondrial inner membrane protein mitofilin controls cristae morphology. Mol Biol Cell 16(3), 1543-1554.

Kamiya, A., Kubo, K.-I., Tomoda, T., Takaki, M., Youn, R., Ozeki, Y., Sawamura, N., Park, U., Kudo, C., Okawa, M., Ross, C.A., Hatten, M.E., Nakajima, K., Sawa, A., 2005. A schizophrenia-associated mutation of DISC1 perturbs cerebral cortex development. Nat Cell Biol 7(12), 1167-1178.

Kamiya, A., Tomoda, T., Chang, J., Takaki, M., Zhan, C., Morita, M., Cascio, M.B., Elashvili, S., Koizumi, H., Takanezawa, Y., Dickerson, F., Yolken, R., Arai, H., Sawa, A., 2006. DISC1-NDEL1/NUDEL protein interaction, an essential component for neurite outgrowth, is modulated by genetic variations of DISC1. Hum Mol Genet 15(22), 3313-3323. Kang, J.S., Tian, J.H., Pan, P.Y., Zald, P., Li, C., Deng, C., Sheng, Z.H., 2008. Docking of axonal mitochondria by syntaphilin controls their mobility and affects short-term facilitation. Cell 132(1), 137-148.

Kim, H.J., Shaker, M.R., Cho, B., Cho, H.M., Kim, H., Kim, J.Y., Sun, W., 2015. Dynaminrelated protein 1 controls the migration and neuronal differentiation of subventricular zonederived neural progenitor cells. Sci Rep 5, 15962.

Kolomeets, N.S., Uranova, N., 2010. Ultrastructural abnormalities of astrocytes in the hippocampus in schizophrenia and duration of illness: A postortem morphometric study. World J Biol Psychiatry 11(2 Pt 2), 282-292.

Kornmann, B., Osman, C., Walter, P., 2011. The conserved GTPase Gem1 regulates endoplasmic reticulum-mitochondria connections. Proc Natl Acad Sci U S A 108(34), 1415114156.

Kulkarni, V.A., Firestein, B.L., 2012. The dendritic tree and brain disorders. Mol Cell Neurosci 50(1), 10-20.

Kung, L., Roberts, R.C., 1999. Mitochondrial pathology in human schizophrenic striatum: a postmortem ultrastructural study. Synapse 31(1), 67-75.

Lee, S., Lee, K.S., Huh, S., Liu, S., Lee, D.Y., Hong, S.H., Yu, K., Lu, B., 2016. Polo Kinase Phosphorylates Miro to Control ER-Mitochondria Contact Sites and Mitochondrial $\mathrm{Ca}(2+)$ Homeostasis in Neural Stem Cell Development. Dev Cell 37(2), 174-189.

Leliveld, S.R., Bader, V., Hendriks, P., Prikulis, I., Sajnani, G., Requena, J.R., Korth, C., 2008. Insolubility of disrupted-in-schizophrenia 1 disrupts oligomer-dependent interactions with nuclear distribution element 1 and is associated with sporadic mental disease. J Neurosci 28(15), 3839-3845.

Levy, M., Faas, G.C., Saggau, P., Craigen, W.J., Sweatt, J.D., 2003. Mitochondrial regulation of synaptic plasticity in the hippocampus. The Journal of Biological Chemistry 278(20), 17727-17734.

Li, Z., Okamoto, K., Hayashi, Y., Sheng, M., 2004. The importance of dendritic mitochondria in the morphogenesis and plasticity of spines and synapses. Cell 119(6), 873-887. 
Lin-Hendel, E.G., McManus, M.J., Wallace, D.C., Anderson, S.A., Golden, J.A., 2016. Differential Mitochondrial Requirements for Radially and Non-radially Migrating Cortical Neurons: Implications for Mitochondrial Disorders. Cell Rep 15(2), 229-237.

Lin, M.T., Beal, M.F., 2006. Mitochondrial dysfunction and oxidative stress in neurodegenerative diseases. Nature 443(7113), 787-795.

Liu, S., Sawada, T., Lee, S., Yu, W., Silverio, G., Alapatt, P., Millan, I., Shen, A., Saxton, W., Kanao, T., Takahashi, R., Hattori, N., Imai, Y., Lu, B., 2012. Parkinson's diseaseassociated kinase PINK1 regulates Miro protein level and axonal transport of mitochondria. PLoS Genet 8(3), e1002537.

Lopez-Domenech, G., Higgs, N.F., Vaccaro, V., Ros, H., Arancibia-Carcamo, I.L., MacAskill, A.F., Kittler, J.T., 2016. Loss of Dendritic Complexity Precedes Neurodegeneration in a Mouse Model with Disrupted Mitochondrial Distribution in Mature Dendrites. Cell Rep 17(2), 317-327.

Lowe, M.T.J., Kim, E.H., Faull, R.L.M., Christie, D.L., Waldvogel, H.J., 2013. Dissociated expression of mitochondrial and cytosolic creatine kinases in the human brain: a new perspective on the role of creatine in brain energy metabolism. J. Cereb. Blood Flow Metab. 33(8), 1295-1306.

MacAskill, A.F., Atkin, T.A., Kittler, J.T., 2010. Mitochondrial trafficking and the provision of energy and calcium buffering at excitatory synapses. The European Journal of Neuroscience 32(2), 231-240.

MacAskill, A.F., Brickley, K., Stephenson, F.A., Kittler, J.T., 2009a. GTPase dependent recruitment of Grif-1 by Miro1 regulates mitochondrial trafficking in hippocampal neurons. Mol Cell Neurosci 40(3), 301-312.

MacAskill, A.F., Kittler, J.T., 2010. Control of mitochondrial transport and localization in neurons. Trends Cell Biol 20(2), 102-112.

Macaskill, A.F., Rinholm, J.E., Twelvetrees, A.E., Arancibia-Carcamo, I.L., Muir, J., Fransson, A., Aspenstrom, P., Attwell, D., Kittler, J.T., 2009b. Miro1 is a calcium sensor for glutamate receptor-dependent localization of mitochondria at synapses. Neuron 61(4), 541555.

Manji, H., Kato, T., Di Prospero, N.A., Ness, S., Beal, M.F., Krams, M., Chen, G., 2012. Impaired mitochondrial function in psychiatric disorders. Nat Rev Neurosci 13(5), 293-307.

McBride, H.M., Neuspiel, M., Wasiak, S., 2006. Mitochondria: more than just a powerhouse. Curr. Biol. 16(14), R551-560.

McCormack, J.G., Halestrap, A.P., Denton, R.M., 1990. Role of calcium ions in regulation of mammalian intramitochondrial metabolism. Physiol Rev 70(2), 391-425.

Millar, J.K., James, R., Christie, S., Porteous, D.J., 2005a. Disrupted in schizophrenia 1 (DISC1): subcellular targeting and induction of ring mitochondria. Mol Cell Neurosci 30(4), 477-484.

Millar, J.K., Pickard, B.S., Mackie, S., James, R., Christie, S., Buchanan, S.R., Malloy, M.P., Chubb, J.E., Huston, E., Baillie, G.S., Thomson, P.A., Hill, E.V., Brandon, N.J., Rain, J.-C., Camargo, L.M., Whiting, P.J., Houslay, M.D., Blackwood, D.H.R., Muir, W.J., Porteous, D.J., 2005b. DISC1 and PDE4B are interacting genetic factors in schizophrenia that regulate cAMP signaling. Science 310(5751), 1187-1191.

Millar, J.K., Wilson-Annan, J.C., Anderson, S., Christie, S., Taylor, M.S., Semple, C.A., Devon, R.S., St Clair, D.M., Muir, W.J., Blackwood, D.H., Porteous, D.J., 2000. Disruption of two novel genes by a translocation co-segregating with schizophrenia. Hum Mol Genet 9(9), 1415-1423.

Miller, K.E., Sheetz, M.P., 2004. Axonal mitochondrial transport and potential are correlated. J Cell Sci 117(Pt 13), 2791-2804. 
Nangaku, M., Sato-Yoshitake, R., Okada, Y., Noda, Y., Takemura, R., Yamazaki, H., Hirokawa, N., 1994. KIF1B, a novel microtubule plus end-directed monomeric motor protein for transport of mitochondria. Cell 79(7), 1209-1220.

Narayan, S., Nakajima, K., Sawa, A., 2013. DISC1: a key lead in studying cortical development and associated brain disorders. Neuroscientist 19(5), 451-464.

Nicholls, D.G., 2009. Mitochondrial calcium function and dysfunction in the central nervous system. Biochim. Biophys. Acta 1787(11), 1416-1424.

Norkett, R., Modi, S., Birsa, N., Atkin, T.A., Ivankovic, D., Pathania, M., Trossbach, S.V., Korth, C., Hirst, W.D., Kittler, J.T., 2016. DISC1-dependent Regulation of Mitochondrial Dynamics Controls the Morphogenesis of Complex Neuronal Dendrites. J Biol Chem 291(2), 613-629.

O'Donovan, M.C., Craddock, N., Norton, N., Williams, H., Peirce, T., Moskvina, V., Nikolov, I., Hamshere, M., Carroll, L., Georgieva, L., Dwyer, S., Holmans, P., Marchini, J.L., Spencer, C.C., Howie, B., Leung, H.T., Hartmann, A.M., Moller, H.J., Morris, D.W., Shi, Y., Feng, G., Hoffmann, P., Propping, P., Vasilescu, C., Maier, W., Rietschel, M., Zammit, S., Schumacher, J., Quinn, E.M., Schulze, T.G., Williams, N.M., Giegling, I., Iwata, N., Ikeda, M., Darvasi, A., Shifman, S., He, L., Duan, J., Sanders, A.R., Levinson, D.F., Gejman, P.V., Cichon, S., Nothen, M.M., Gill, M., Corvin, A., Rujescu, D., Kirov, G., Owen, M.J., Buccola, N.G., Mowry, B.J., Freedman, R., Amin, F., Black, D.W., Silverman, J.M., Byerley, W.F., Cloninger, C.R., Molecular Genetics of Schizophrenia, C., 2008. Identification of loci associated with schizophrenia by genome-wide association and followup. Nat Genet 40(9), 1053-1055.

Ogawa, F., Malavasi, E.L., Crummie, D.K., Eykelenboom, J.E., Soares, D.C., Mackie, S., Porteous, D.J., Millar, J.K., 2014. DISC1 complexes with TRAK1 and Miro1 to modulate anterograde axonal mitochondrial trafficking. Hum Mol Genet 23(4), 906-919.

Ogawa, F., Murphy, L.C., Malavasi, E.L., O'Sullivan, S.T., Torrance, H.S., Porteous, D.J., Millar, J.K., 2016. NDE1 and GSK3beta Associate with TRAK1 and Regulate Axonal Mitochondrial Motility: Identification of Cyclic AMP as a Novel Modulator of Axonal Mitochondrial Trafficking. ACS Chem Neurosci 7(5), 553-564.

Park, C., Lee, S.A., Hong, J.H., Suh, Y., Park, S.J., Suh, B.K., Woo, Y., Choi, J., Huh, J.W., Kim, Y.M., Park, S.K., 2016. Disrupted-in-schizophrenia 1 (DISC1) and Syntaphilin collaborate to modulate axonal mitochondrial anchoring. Mol Brain 9(1), 69.

Park, S.J., Jeong, J., Park, Y.U., Park, K.S., Lee, H., Lee, N., Kim, S.M., Kuroda, K., Nguyen, M.D., Kaibuchi, K., Park, S.K., 2015. Disrupted-in-schizophrenia-1 (DISC1) Regulates Endoplasmic Reticulum Calcium Dynamics. Sci Rep 5, 8694.

Park, Y.-U., Jeong, J., Lee, H., Mun, J.Y., Kim, J.-H., Lee, J.S., Nguyen, M.D., Han, S.S., Suh, P.-G., Park, S.K., 2010. Disrupted-in-schizophrenia 1 (DISC1) plays essential roles in mitochondria in collaboration with Mitofilin. Proc Natl Acad Sci U S A 107(41), 1778517790.

Pickrell, A.M., Youle, R.J., 2015. The roles of PINK1, parkin, and mitochondrial fidelity in Parkinson's disease. Neuron 85(2), 257-273.

Pilling, A.D., Horiuchi, D., Lively, C.M., Saxton, W.M., 2006. Kinesin-1 and Dynein are the primary motors for fast transport of mitochondria in Drosophila motor axons. Mol Biol Cell 17(4), 2057-2068.

Pinero-Martos, E., Ortega-Vila, B., Pol-Fuster, J., Cisneros-Barroso, E., Ruiz-Guerra, L., Medina-Dols, A., Heine-Suner, D., Llado, J., Olmos, G., Vives-Bauza, C., 2016. Disrupted in Schizophrenia 1 (DISC1) is a constituent of the mammalian Mitochondrial contact site and Cristae Organizing System (MICOS) complex, and is essential for oxidative phosphorylation. Hum Mol Genet. 
Quintero, O.A., DiVito, M.M., Adikes, R.C., Kortan, M.B., Case, L.B., Lier, A.J., Panaretos, N.S., Slater, S.Q., Rengarajan, M., Feliu, M., Cheney, R.E., 2009. Human Myo19 is a novel myosin that associates with mitochondria. Curr Biol 19(23), 2008-2013.

Roberts, R.C., Barksdale, K.A., Roche, J.K., Lahti, A.C., 2015. Decreased synaptic and mitochondrial density in the postmortem anterior cingulate cortex in schizophrenia. Schizophr Res 168(1-2), 543-553.

Rossignol, D.A., Frye, R.E., 2012. Mitochondrial dysfunction in autism spectrum disorders: a systematic review and meta-analysis. Mol Psychiatry 17(3), 290-314.

Rowland, A.A., Voeltz, G.K., 2012. Endoplasmic reticulum-mitochondria contacts: function of the junction. Nat Rev Mol Cell Biol 13(10), 607-625.

Sachs, N.A., Sawa, A., Holmes, S.E., Ross, C.A., DeLisi, L.E., Margolis, R.L., 2005. A frameshift mutation in Disrupted in Schizophrenia 1 in an American family with schizophrenia and schizoaffective disorder. Mol Psychiatry 10(8), 758-764.

Santini, E., Turner, K.L., Ramaraj, A.B., Murphy, M.P., Klann, E., Kaphzan, H., 2015. Mitochondrial Superoxide Contributes to Hippocampal Synaptic Dysfunction and Memory Deficits in Angelman Syndrome Model Mice. The Journal of Neuroscience: The Official Journal of the Society for Neuroscience 35(49), 16213-16220.

Seshadri, S., Kamiya, A., Yokota, Y., Prikulis, I., Kano, S., Hayashi-Takagi, A., Stanco, A., Eom, T.Y., Rao, S., Ishizuka, K., Wong, P., Korth, C., Anton, E.S., Sawa, A., 2010. Disrupted-in-Schizophrenia-1 expression is regulated by beta-site amyloid precursor protein cleaving enzyme-1-neuregulin cascade. Proc Natl Acad Sci U S A 107(12), 5622-5627.

Shao, C.Y., Zhu, J., Xie, Y.J., Wang, Z., Wang, Y.N., Wang, Y., Su, L.D., Zhou, L., Zhou, T.H., Shen, Y., 2013. Distinct functions of nuclear distribution proteins LIS1, Ndel1 and NudCL in regulating axonal mitochondrial transport. Traffic 14(7), 785-797.

Shao, L., Martin, M.V., Watson, S.J., Schatzberg, A., Akil, H., Myers, R.M., Jones, E.G., Bunney, W.E., Vawter, M.P., 2008. Mitochondrial involvement in psychiatric disorders. Ann Med 40(4), 281-295.

Sheng, Z.H., Cai, Q., 2012. Mitochondrial transport in neurons: impact on synaptic homeostasis and neurodegeneration. Nat Rev Neurosci 13(2), 77-93.

Singh, K.K., De Rienzo, G., Drane, L., Mao, Y., Flood, Z., Madison, J., Ferreira, M., Bergen, S., King, C., Sklar, P., Sive, H., Tsai, L.H., 2011. Common DISC1 polymorphisms disrupt Wnt/GSK3beta signaling and brain development. Neuron 72(4), 545-558.

St Clair, D., Blackwood, D., Muir, W., Carothers, A., Walker, M., Spowart, G., Gosden, C., Evans, H.J., 1990. Association within a family of a balanced autosomal translocation with major mental illness. Lancet 336(8706), 13-16.

Stephen, T.L., Gupta-Agarwal, S., Kittler, J.T., 2014. Mitochondrial dynamics in astrocytes. Biochem Soc Trans 42(5), 1302-1310.

Stephen, T.L., Higgs, N.F., Sheehan, D.F., Al Awabdh, S., Lopez-Domenech, G., ArancibiaCarcamo, I.L., Kittler, J.T., 2015. Miro1 Regulates Activity-Driven Positioning of Mitochondria within Astrocytic Processes Apposed to Synapses to Regulate Intracellular Calcium Signaling. J Neurosci 35(48), 15996-16011.

Sung, J.Y., Engmann, O., Teylan, M.A., Nairn, A.C., Greengard, P., Kim, Y., 2008. WAVE1 controls neuronal activity-induced mitochondrial distribution in dendritic spines. Proc Natl Acad Sci U S A 105(8), 3112-3116.

Szabadkai, G., Duchen, M.R., 2008. Mitochondria: the hub of cellular Ca2+ signaling. Physiology (Bethesda) 23, 84-94.

Takeuchi, T., Duszkiewicz, A.J., Morris, R.G.M., 2013. The synaptic plasticity and memory hypothesis: encoding, storage and persistence. Philosophical Transactions of the Royal Society B: Biological Sciences 369(1633), 20130288-20130288. 
Taya, S., Shinoda, T., Tsuboi, D., Asaki, J., Nagai, K., Hikita, T., Kuroda, S., Kuroda, K., Shimizu, M., Hirotsune, S., Iwamatsu, A., Kaibuchi, K., 2007. DISC1 regulates the transport of the NUDEL/LIS1/14-3-3epsilon complex through kinesin-1. J Neurosci 27(1), 15-26.

Thomson, P.A., Parla, J.S., McRae, A.F., Kramer, M., Ramakrishnan, K., Yao, J., Soares, D.C., McCarthy, S., Morris, S.W., Cardone, L., Cass, S., Ghiban, E., Hennah, W., Evans, K.L., Rebolini, D., Millar, J.K., Harris, S.E., Starr, J.M., MacIntyre, D.J., Generation, S., McIntosh, A.M., Watson, J.D., Deary, I.J., Visscher, P.M., Blackwood, D.H., McCombie, W.R., Porteous, D.J., 2014. 708 Common and 2010 rare DISC1 locus variants identified in 1542 subjects: analysis for association with psychiatric disorder and cognitive traits. Mol. Psychiatry 19(6), 668-675.

Tomppo, L., Hennah, W., Miettunen, J., Jarvelin, M.R., Veijola, J., Ripatti, S., Lahermo, P., Lichtermann, D., Peltonen, L., Ekelund, J., 2009. Association of variants in DISC1 with psychosis-related traits in a large population cohort. Arch Gen Psychiatry 66(2), 134-141.

Trossbach, S.V., Bader, V., Hecher, L., Pum, M.E., Masoud, S.T., Prikulis, I., Schable, S., de Souza Silva, M.A., Su, P., Boulat, B., Chwiesko, C., Poschmann, G., Stuhler, K., Lohr, K.M., Stout, K.A., Oskamp, A., Godsave, S.F., Muller-Schiffmann, A., Bilzer, T., Steiner, H., Peters, P.J., Bauer, A., Sauvage, M., Ramsey, A.J., Miller, G.W., Liu, F., Seeman, P., Brandon, N.J., Huston, J.P., Korth, C., 2016. Misassembly of full-length Disrupted-inSchizophrenia 1 protein is linked to altered dopamine homeostasis and behavioral deficits. Mol Psychiatry.

Tsuboi, D., Kuroda, K., Tanaka, M., Namba, T., Iizuka, Y., Taya, S., Shinoda, T., Hikita, T., Muraoka, S., Iizuka, M., Nimura, A., Mizoguchi, A., Shiina, N., Sokabe, M., Okano, H., Mikoshiba, K., Kaibuchi, K., 2015. Disrupted-in-schizophrenia 1 regulates transport of ITPR1 mRNA for synaptic plasticity. Nat Neurosci 18(5), 698-707.

van Spronsen, M., Mikhaylova, M., Lipka, J., Schlager, M.A., van den Heuvel, D.J., Kuijpers, M., Wulf, P.S., Keijzer, N., Demmers, J., Kapitein, L.C., Jaarsma, D., Gerritsen, H.C., Akhmanova, A., Hoogenraad, C.C., 2013. TRAK/Milton motor-adaptor proteins steer mitochondrial trafficking to axons and dendrites. Neuron 77(3), 485-502.

Vos, M., 2010. Synaptic mitochondria in synaptic transmission and organization of vesicle pools in health and disease. Frontiers in Synaptic Neuroscience 2.

Wang, X., Schwarz, T.L., 2009. The mechanism of Ca2+ -dependent regulation of kinesinmediated mitochondrial motility. Cell 136(1), 163-174.

Wang, X., Winter, D., Ashrafi, G., Schlehe, J., Wong, Y.L., Selkoe, D., Rice, S., Steen, J., LaVoie, M.J., Schwarz, T.L., 2011. PINK1 and Parkin target Miro for phosphorylation and degradation to arrest mitochondrial motility. Cell 147(4), 893-906.

Wen, Z., Nguyen, H.N., Guo, Z., Lalli, M.A., Wang, X., Su, Y., Kim, N.S., Yoon, K.J., Shin, J., Zhang, C., Makri, G., Nauen, D., Yu, H., Guzman, E., Chiang, C.H., Yoritomo, N., Kaibuchi, K., Zou, J., Christian, K.M., Cheng, L., Ross, C.A., Margolis, R.L., Chen, G., Kosik, K.S., Song, H., Ming, G.L., 2014. Synaptic dysregulation in a human iPS cell model of mental disorders. Nature 515(7527), 414-418.

Werth, J.L., Thayer, S.A., 1994. Mitochondria buffer physiological calcium loads in cultured rat dorsal root ganglion neurons. The Journal of Neuroscience: The Official Journal of the Society for Neuroscience 14(1), 348-356.

Wondolowski, J., Dickman, D., 2013. Emerging links between homeostatic synaptic plasticity and neurological disease. Frontiers in Cellular Neuroscience 7.

Zhou, X., Chen, Q., Schaukowitch, K., Kelsoe, J.R., Geyer, M.A., 2010. Insoluble DISC1Boymaw fusion proteins generated by DISC1 translocation. Mol Psychiatry.

Zhou, X., Geyer, M.A., Kelsoe, J.R., 2008. Does disrupted-in-schizophrenia (DISC1) generate fusion transcripts? Mol Psychiatry 13(4), 361-363. 
Zucker, R.S., 1999. Calcium- and activity-dependent synaptic plasticity. Curr. Opin. Neurobiol. 9(3), 305-313. 


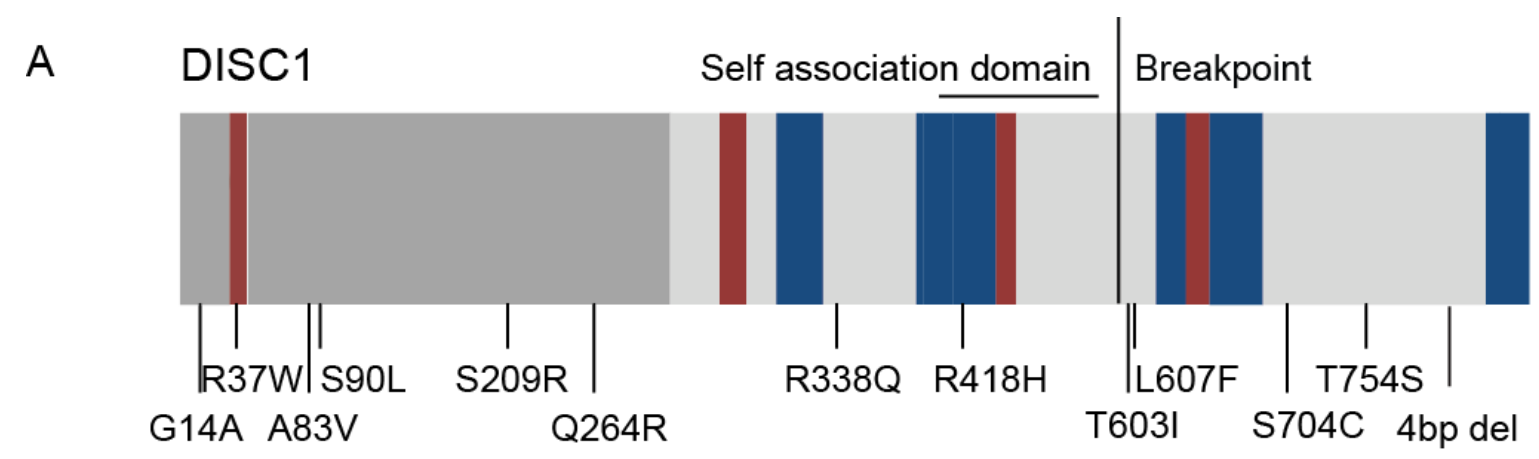

B DISC1-Boymaw fusion protein

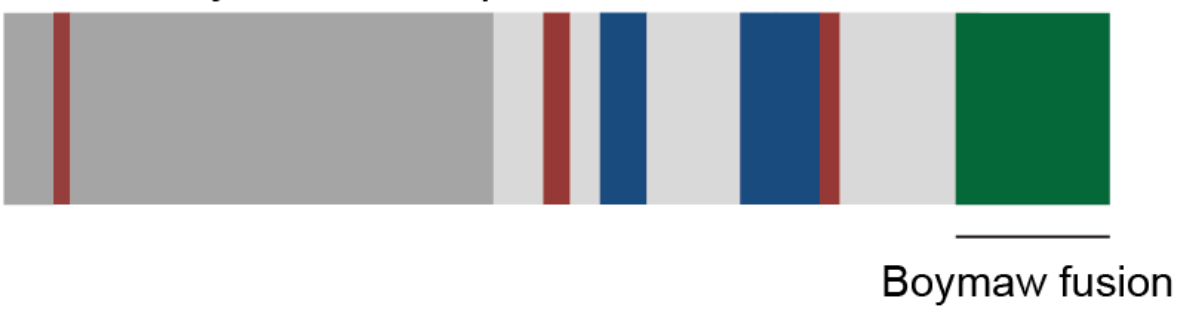

DISC1 truncation

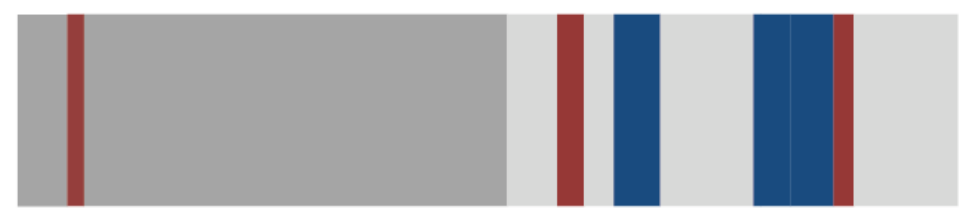

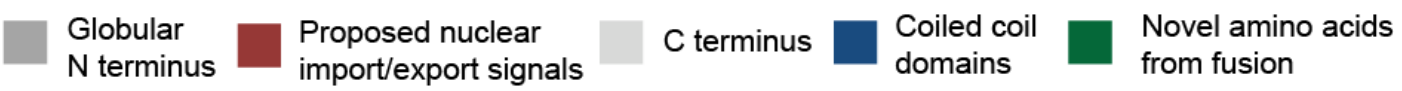

Figure 1: Schematic of the DISC1 protein. A Structure of the DISC1 protein showing globular $\mathrm{N}$ terminus, Coiled coil rich $\mathrm{C}$ terminus and sites of psychiatric disease associated mutations. B Possible outcomes of the balanced chromosomal translocation at the protein level. The translocation may give rise to a fusion protein between DISC1 and Boymaw/DISC1 fusion partner 1, or a truncated DISC1. 
A - Wild type DISC1

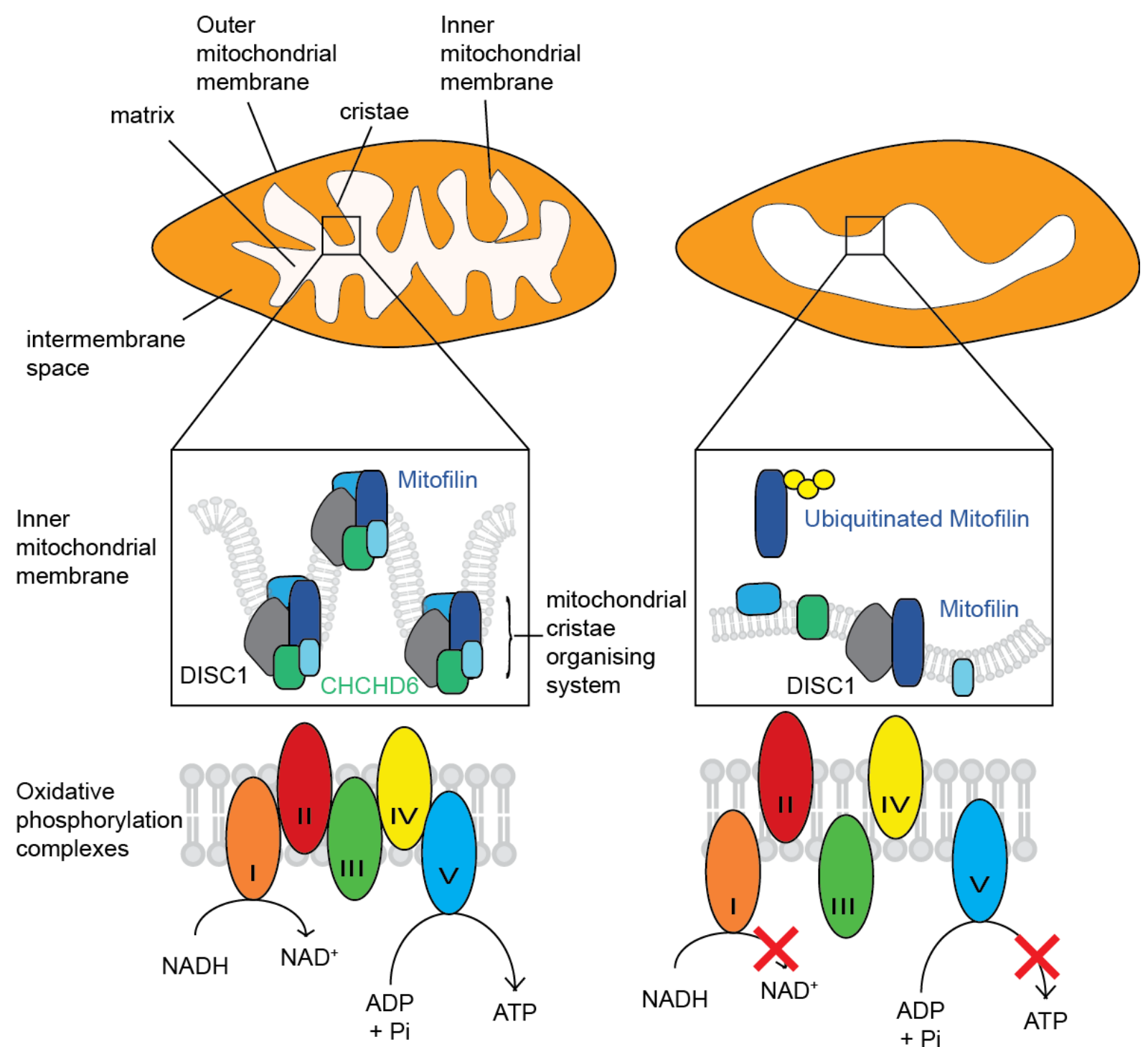

Figure 2: DISC1 as a regulator of mitochondrial function. A DISC1 interacts with mitofilin and other components of mitochondrial cristae organising complexes to correctly fold the inner mitochondrial membrane. Under these conditions oxidative phosphorylation for ATP production proceeds normally. B Loss of wild type DISC1 leads to collapse of cristae via mitofilin degradation and disassembly of cristae organising complexes. Under these conditions, mitochondrial membrane potential is decreased and so, ATP production is impaired. 
A

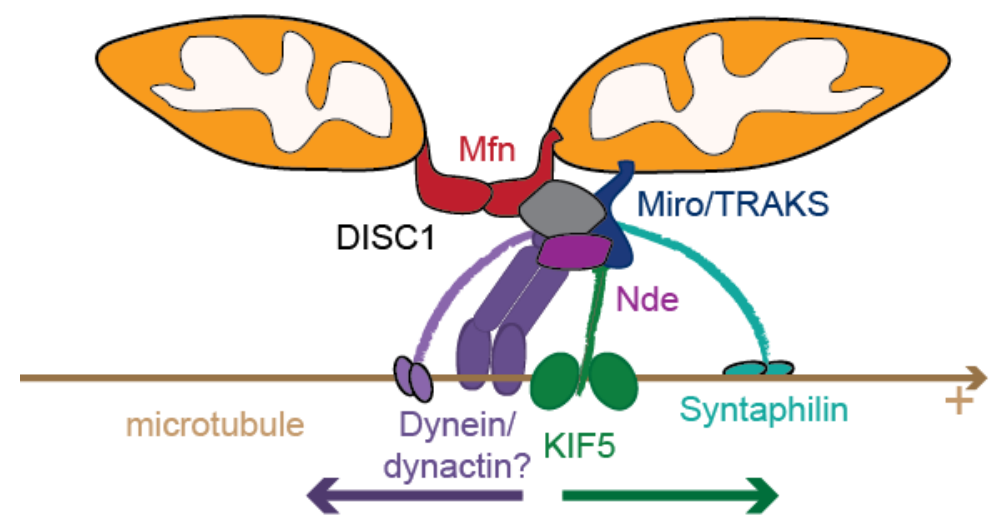

B

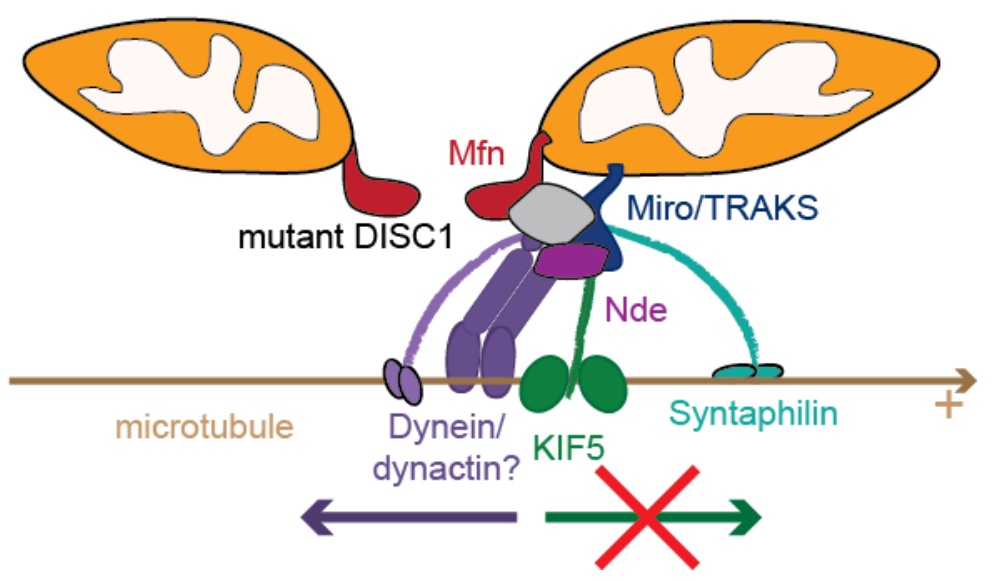

Figure 3: DISC1 as a regulator of mitochondrial trafficking. A DISC1 interacts with molecular motor complexes (kinesin and dynein) known to be essential for microtubule based transport of mitochondria. DISC1 also interacts with Miro, TRAK and syntaphilin proteins to specifically regulate mitochondrial transport, and mitofusins to regulate mitochondrial fusion. B Mutant DISC1 impairs microtubule based transport of mitochondria with kinesin mediated anterograde transport more affected. 